\title{
X-ray Nanoscope for Electronics Assurance
}

Harry E. Martz, Jr., Jeffrey S. Kallman, Charles J. Divin and Stephen Azevedo Lawrence Livermore National Laboratory

Livermore, CA 94551

\author{
August 23, 2013 \\ LLNL-TR-641601 \\ (IM\# 760141)
}

Version \#8

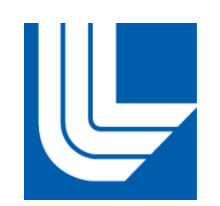


This document was prepared as an account of work sponsored by an agency of the United States government. Neither the United States government nor Lawrence Livermore National Security, LLC, nor any of their employees, makes any warranty, expressed or implied, or assumes any legal liability or responsibility for the accuracy, completeness, or usefulness of any information, apparatus, product, or process disclosed, or represents that its use would not infringe privately owned rights. Reference herein to any specific commercial product, process, or service by trade name, trademark, manufacturer, or otherwise does not necessarily constitute or imply its endorsement, recommendation, or favoring by the United States government or Lawrence Livermore National Security, LLC. The views and opinions of authors expressed herein do not necessarily state or reflect those of the United States government or Lawrence Livermore National Security, LLC, and shall not be used for advertising or product endorsement purposes.

This work performed under the auspices of the U.S. Department of Energy by Lawrence Livermore National Laboratory under Contract DE-AC52-07NA27344. 


\title{
X-ray Nanoscope for Electronics Assurance
}

\author{
Harry E. Martz, Jr., Jeffrey S. Kallman, Charles J. Divin, and Stephen Azevedo \\ P.O. Box 808, L-151 \\ Lawrence Livermore National Laboratory \\ Livermore, CA 94551 \\ martz2@llnl.gov
}

\section{Background}

The United States does not have a comprehensive program to certify that integrated circuits (ICs) going into U.S. weapons systems do not contain malicious circuits. Destructive techniques are slow and labor intensive. In response to these concerns, DARPA initiated its TRUST in Integrated Circuits program to develop nondestructive technologies that will ensure the trust of ICs used in military systems, but designed and fabricated under untrusted conditions.

One of the techniques investigated under the DARPA TRUST program was the use of Xray computed tomography (CT) to validate the circuitry on an integrated circuit [1]. As part of the program, DARPA purchased an Xradia UltraXRM-S system ${ }^{1}$ for use as a CT imaging system connected to a synchrotron $X$-ray source. We refer to the DARPA UltraXRM-S as the DARPA or X-ray nanoscope. The nanoscsope is capable of nondestructive 3D imaging of buried nanostructures, and the accurate measurement of voids, cracks, defects, porosity, and particle distributions. For chip analysis, the tunable $\mathrm{x}$-ray energy enhances the material discrimination of metallic $\mathrm{Cu}$ and $\mathrm{Al}$ traces, while maintaining sufficient energy to penetrate the circuit substrates, eliminating the need for conventional destructive measurements.

When the TRUST program funding ended, the DARPA nanoscope was shipped to the Lawrence Livermore National Laboratory (LLNL) in summer 2013 (See Appendix A for the reasons). In addition to building upon the DARPA TRUST program and performing chip imaging, LLNL will use this high resolution X-ray instrument to perform studies of the fine structure of materials and other devices in 3D. This will enable LLNL to: (1) image electronic and MEMS chips for verification of function; (2) characterize the performance of high explosives as a function of voids; (3) better understand the processes used to manufacture very low density foams and (4) help the National Ignition Facility better understand their materials and targets.

This document provides estimates on the expected performance of the DARPA X-ray nanoscope for chip inspection, and LLNL's plans to achieve these goals.

\footnotetext{
${ }^{1}$ The DARPA system was customized for chip inspection and does not have the full feature set of an UltraXRM-S system.
} 


\section{Nanoscope Instrument Characteristics}

The DARPA nanoscope is a modified version of Xradia's imaging product for synchrotrons, as shown in Table 1. It is optimized for electronics assurance and provides a subset (Column 2) of the full UltraXRM-S features (Column 3), though it can be upgraded/extended to include the full features. The DARPA nanoscope optics are optimized for operation around $9 \mathrm{keV}$, which is necessary for penetrating thick substrates and imaging the high atomic weight elements commonly found in integrated circuits.

Table 1. Comparison of the DARPA nanoscope (Current) and possible upgrades (Upgrade).

\begin{tabular}{|l|c|c|}
\hline \multicolumn{1}{|c|}{ Current } & Upgrade \\
\hline Model Name & DARPA X-ray nanoscope & UltraXRM-S200 \\
\hline Imaging Modes & $\begin{array}{c}8-11 \mathrm{keV} \text { Absorption } \\
\text { (optimized for 9 keV) }\end{array}$ & $\begin{array}{c}5-11 \mathrm{keV} \text { Absorption } \\
5-11 \mathrm{keV} \text { Phase Contrast }\end{array}$ \\
\hline Sample Stage & $\begin{array}{c}\mathrm{XYZ}( \pm 5 \mathrm{~mm} \text { travel }) \\
\Theta \quad\left( \pm 135^{\circ} \text { travel }\right)\end{array}$ & [same] \\
\hline Available Magnifications & Mag 1: 600X & $\begin{array}{c}\text { Mag 1: 600X } \\
\text { Mag 2: 1200X }\end{array}$ \\
\hline
\end{tabular}

The key differences lie in the wider energy range and the higher magnification available with the upgraded system. Table 2 compares the estimated 2D radiography and 3D tomography differences between the two magnification values (600X and 1200X) for the 8 to $11 \mathrm{keV}$ energy range. As with all CT systems, users must trade off spatial resolution, inspection volume and imaging time.

Table 2. Imaging performance in the $8-11 \mathrm{keV}$ range.

\begin{tabular}{|c|c|c|}
\hline & Magnification 1 & Magnification 2 \\
\hline 2D Digital Radiography & & \\
\hline Spatial resolution & $50 \mathrm{~nm}$ & $30 \mathrm{~nm}$ \\
\hline Pixel size & $20 \mathrm{~nm}$ & $10 \mathrm{~nm}$ \\
\hline $\begin{array}{l}\text { Field of view: } \\
\text { single tile } \\
\text { mosaic }\end{array}$ & $\begin{aligned} 40 & \times 40 \mu \mathrm{m} \\
1 & \times 1 \mathrm{~cm}\end{aligned}$ & $\begin{aligned} 20 & \times 20 \mu \mathrm{m} \\
1 & \times 1 \mathrm{~cm}\end{aligned}$ \\
\hline Depth of focus & $\pm 40 \mu \mathrm{m}$ & $\pm 20 \mu \mathrm{m}$ \\
\hline 3D X-ray Tomography & & \\
\hline $\begin{array}{l}\text { \# of 2D projections required for a } \\
\text { single 3D volume }\end{array}$ & \multicolumn{2}{|c|}{100 projections } \\
\hline Time between projections & \multicolumn{2}{|c|}{$<2 \mathrm{~s}$} \\
\hline $\begin{array}{l}\text { Reconstructed volume } \\
0 \% \text { horizontal overlap } \\
\text { 30\% overlap (for mosaic scan) }\end{array}$ & $\begin{array}{l}38 \times 38 \times 38 \mu^{3} \\
27 \times 27 \times 38 \mu \mathrm{m}^{3} \\
\end{array}$ & $\begin{array}{l}18 \times 18 \times 18 \mu \mathrm{m}^{3} \\
14 \times 14 \times 18 \mathrm{~m}^{3}\end{array}$ \\
\hline Voxel size & $(20 \mathrm{~nm})^{3}$ & $(10 \mathrm{~nm})^{3}$ \\
\hline Typical acq. time for $0 \%$ overlap & \multicolumn{2}{|c|}{$\sim 3$ minutes } \\
\hline
\end{tabular}




\section{Estimated Performance for Electronics Assurance}

For the purpose of electronic assurance under the TRUST program, work has already been performed using X-ray nanoCT by Michael Bajura at USC [1,2]. Using a prototype instrument at the SLAC National Accelerator Laboratory at Stanford, a great deal was learned about acquiring the projection data for CT, integration time per image, stitching together projections, image processing, reconstruction, etc. The chip used for this early work was extracted from its carrier and back-thinned in order to reduce the data acquisition time.

One issue with the use of the nanoCT as configured at SLAC was the predicted long data acquisition times for a chip, approximately 1000 days. This estimate was based on prototype hardware and basic reconstruction algorithms, which require many 2D projections to obtain a 3D volume. For example, with the 3.5-sec projection time on the SLAC systems, the time to acquire a full 3D scan of a large chip of $2-\mathrm{cm}$ per side $\left(4 \mathrm{~cm}^{2}\right.$ area) would be about six years! (Note that Bajura's estimate was for a smaller chip with an area of $2 \mathrm{~cm}^{2}$.)

$$
\begin{aligned}
\text { Time } & =\frac{\text { Time }}{\text { projection }} \times \frac{\# \text { projections }}{\text { CT subvolume }} \times \frac{\text { chip area }}{\text { subvolume area }} \\
& =(3.5 \mathrm{~s}) \times(100) \times \frac{(2 \mathrm{~cm})^{2}}{(27 \mu \mathrm{m})^{2}}=6 \text { years }
\end{aligned}
$$

This time estimate for scanning a single chip is clearly unreasonable. However, there are several factors that can greatly reduce inspection times to match those of destructive techniques, without destroying the chip in the process. Some of those ideas are to

- Speed-up the projection time - The time for a single projection, and the motion control to move to the next projection, could be sped up by a factor of 2 .

- Scan sub-volumes instead of the full chip - There are often suspect areas of much small volume that can be the focus of the scan.

- Employ limited-data CT techniques - There is a healthy literature base, and our group has experience, in reconstructing CT volumes from fewer views.

Our future plans are to employ all of these techniques to reduce this data acquisition and processing time. For example, by combining the UltraXRM's faster acquisition with the algorithmic improvements to reconstruct with only 10 views will significantly reduce the time required to image a full chip to four months.

$$
=(2 s) \times(10) \times \frac{(2 \mathrm{~cm})^{2}}{(27 \mu \mathrm{m})^{2}}=4 \text { months }
$$

The more normal case will be to focus our scanning on a smaller chip area of interest, which will likely be on the order of $0.5 \mathrm{~cm}$ and could be scanned in eight days.

$$
=(2 \mathrm{~s}) \times(10) \times \frac{(0.5 \mathrm{~cm})^{2}}{(27 \mu \mathrm{m})^{2}}=8 \text { days }
$$

These types of scan times would make the use of the DARPA nanoscope for electronics assurance a more feasible option. 


\section{LLNL Future Plans}

We are pursuing funding to develop other methods to reduce data acquisition and processing times, including noise reduction and limited angle CT [3,4], and ptychography (coherent diffraction imaging) [5]. However, the main technical hurdle is to make the DARPA nanoscope operational. A separate plan has been developed to install the nanoscope on an unused beam-line at the Advanced Light Source (ALS) at Lawrence Berkeley National Laboratory.

The following work is needed to make the nanoscope operational and could be completed in one year if the following efforts are funded:

1. Develop a consortium of end users to provide funds to be able to upgrade an ALS beam-line to enable the nanoscope to be installed.

2. Select and finalize design of ALS beam line.

3. Construct the upgraded ALS beam line.

4. Ship nanoscope to ALS.

5. Install and commission the nanoscope.

6. Reduce-to-practice the methods needed for faster data acquisition, such as limited angle image reconstruction algorithms and ptychography.

More details about the scope, budget and deliverables of this project are available upon request.

\section{References}

[1] M. Bajura, et al., "Imaging Integrated Circuits with X-ray Microscopy,” Proc. of the $36^{\text {th }}$ GOMACTech Conference, Orlando, FL, March 2011.

[2] M. Bajura, et al., "Verification of integrated circuits against malicious circuit insertions and modifications using non-destructive x-ray microscopy,” U. S. Patent \#8,139,846, March 20, 2012.

[3] E. Y. Sidky and C.-M. Kao and X. Pan, "Accurate image reconstruction from fewviews and limited-angle data in divergent-beam CT," J. X-ray Sci. Tech., vol. 14, pp. 119-139, 2006.

[4] Zhiqiang Chen, Xin Jin, Liang Li, and Ge Wang, “A limited-angle CT reconstruction method based on anisotropic TV minimization”, Phys. Med. \& Biol., vol. 58, pp. 2119 - 2141, 2013.

[5] A. M. Maiden, M. J. Humphry, and J. M. Rodenburg, "Ptychographic transmission microscopy in three dimensions using a multi-slice approach,” J. Opt. Soc. Am. A, Vol. 29, No. 8, pp.1606-1614, August 2012. 


\title{
6 Appendix A: Email from DARPA Program Manager on 4/10/2013 regarding the final disposition of the Nano-CT instrument
}

\begin{abstract}
Gentlemen -
Thank you for the time and travel you invested to help DARPA determine the best disposition the XRADIA tool built for the TRUST Program. Any one of the proposed homes would fully leverage the capabilities of the tool, but based on the meeting and subsequent discussions, it becomes quite apparent that Lawrence Livermore National Laboratory is the most appropriate home. The approach to the decision was analytic, and was anything but whimsical or capricious. Here were the considerations, using the criteria you were already familiar with:
\end{abstract}

AVAILABLE ILLUMINATION SOURCE: It was not yet clear if alternative XRAY sources under consideration are sufficient for developing the $9 \mathrm{KeV}$ illumination intensity required by the tool. There is an X-ray beam-line at ALS that can be dedicated to this immediately (LLNL indicates it was actually built for the semiconductor industry); much of the infrastructure is already there, including power, AC, etc. Xradia will be pulled into the install, but it will not be used until the beam-line is (re-)commissioned. This arrangement will be optimal, as the device will be "useroperated" with exclusive LLNL / partner access whenever needed. LLNL will be exploring the funding to make this happen. None of the other site options provide this flexibility and assurance in being able to execute the mission. DMEA had a plan to determine the right source; that proposal however was the most risky since presently no XRAY source resides at DMEA. FUNDING AND SPACE: Space was pretty much a draw for all locations. All sites had space. The labs did not have a chance yet to respond with budget accommodation. DMEA funding was dependent in part upon a multi-phase SBIR. It was not apparent that DMEA had assured extended funding which covered retooling, source purchase, ongoing operation, maintenance, and training, given that their PH II funds plus the add-on would already be fully devoted to cover installation. A budget already exists at LLNL for the support of 3 existing tools, which would need to be extended to 4 .

SITE AND STAFF EXPERIENCE: LLNL was again the clear preference here - 3 Xradia tools are already installed and running at LLNL; years of CT R\&D; depth of knowledge by an experienced staff, and critical mass in tool maintenance and modification. No fundamental training was necessary. Support would also be available by Dan Schneberk, and Chuck Divin who is the primary user of the Xradia UltraXRM (NanoCT) system at LLNL. James Hunter and Bill Ward (LANL), and Enrico Katana and Kyle Thompson (Sandia) were also recognized for their prowess. It is assumed that they will be available for consultation if the need arises at LLNL. DMEA has no previous experience with the $X$-ray tool. They indicated that they would require training and funded support from Xradia; no backup support was identified in their proposal.

INTENDED APPLICATION: LLNL is perhaps not as immersed in the specific security application that our community focused on; this consideration favored DMEA as best choice. Subsequent discussions with LLNL, however, assured me that their site mission includes both research and operation. The other labs also would have offered this. DMEA's proposal envisioned solely an RE production imaging use; however it was made clear in the discussion that more work with this tool is needed before it can be considered to be ready for prime time RE. It is not yet a turn-key RE solution. 
COMMUNITY ACCESS: LLNL offers unclass access in a university environment. CONOPS and Protocols will need to be developed to maintain their opsec however and to accommodate the university culture. Bending magnets installed at that location will provide necessary beam access for multiple experiments. Further, LLNL leadership assures me that tool access will be extended to the other labs and to DMEA. DMEA indicated that they would provide limited access to the user community when it is up and running due to their desire to use the tool in a production environment. Labs other than LLNL and DMEA all placed the device in a restricted access setting.

TIME TO "UP AND RUNNING": It was anticipated (and later confirmed in subsequent discussion) that the labs in general, and LLNL specifically would be able to initiate installation of the tool upon receipt, and put it to use as soon as it was up and running. DMEA proposal indicated that it would be greater than or equal to 2 years before the asset would be operational, and would depend upon XRADIA retrofit and personnel training. The labs present no such impediment.

CONCERNS: We trust that XRADIA will still honor the terms of the sale agreement, which called for it to cover shipping and 1 week of on-site product support. It's certainly in their best interests to get this tool out and running. The other concern is that LLNL has not yet had time to incorporate the additional expense into their budget - internal R\&D (LDRD) at LLNL or LBNL, direct funding from NSA, and other sponsors using current machines are likely paths for LLNL. Given that 3 other tools are already funded and supported at ALS, we do not anticipate that this will be a showstopper. If so, DARPA will revisit the decision.

The net is that LLNL is identified by DARPA as the final recipient of the TRUST Program's XRADIA tool, using an objective comparison of multiple important criteria. LLNL POCs are Harry Martz and Steve Azevedo.

I request Mike Bajura and Saverio Fazzari to work with XRADIA to affect the transfer of this tool to Lawrence Livermore National Laboratories in as timely a manner as soon as possible, to fulfill the 15 April boundary condition.

Again, I want to thank everyone involved for their responsiveness and their support of this analysis.

Kerry Bernstein, Program Manager

Microsystems Technology Office

Defense Advanced ResearchProjects Agency (DARPA) Kerry.bernstein@darpa.mil

Kerry.bernstein@darpa.smil.mil Kerry.bernstein@darpa.ic.gov

(703) 526-2117 (commercial)

984-0337 (secure) 